\title{
Robust Automatic Coregistration, Segmentation, and Classification of Cell Nuclei in Multimodal Cytopathological Microscopic Images
}

\author{
Thomas Würflinger ${ }^{\text {a }}$, Jens Stockhausen ${ }^{\mathrm{a}}$, \\ Dietrich Meyer-Ebrecht ${ }^{\mathrm{a}}$ and Alfred Böcking ${ }^{\mathrm{b}}$ \\ ${ }^{\mathrm{a}}$ Institute for Measurement Techniques and Image Processing, RWTH Aachen, \\ Germany \\ ${ }^{\mathrm{b}}$ Institute for Cytopathology, Heinrich-Heine-University, Düsseldorf, Germany
}

\begin{abstract}
The paper describes the key component of the Multimodal Cell Analysis approach, a novel cytologic evaluation method for early cancer detection. The approach is based on repeated staining of a cell smear. The correlation of features and data extracted from the different stains, and related to relocated individual cells, may yield a dramatic increase of diagnostic reliability.

In order to utilise the technique, fully automatic, adaptive image preprocessing techniques need to be applied, which are described in this article: coregistration of multimodal images, segmentation, and classification of cell nuclei. The presented feasibility study shows both efficiency and robustness of all steps being high regarding medical image material, and it strongly supports clinical application.
\end{abstract}

Key words: cytopathology, cancer diagnostics, cell microscopy, multimodal coregistration, image segmentation, active contour models, classification.

\section{Introduction}

Different approaches to cancer diagnostics may be distinguished according to the order of magnification at which diagnostic information is retrieved:

\section{Macroscopic Pathology}

Morphologic as well as functional studies, sometimes with additional tissue 
characterisation, can be performed non-invasively by means of modern diagnostic imaging modalities such as CT, MR, fMR, PET, SPECT or sonography. Tumours must have grown up to a considerable size, however, to be recognised.

\section{Histopathology}

The widely applied histologic investigations can detect cancer on a microscopic level and thus earlier. But they require tissue specimens and therefore necessitate more elaborate invasive procedures. If not acquired in the course of an ongoing operation, a biopsy has to be performed, which is bloody, painful and expensive.

\section{Cytopathology}

In contrast, cytopathologic investigations only require cells, which will be obtained non-invasively and without discomfort for the patient. Sediments from diverse body fluids, smears from mucosal surfaces or fine needle aspirates from different organs mostly contain enough cells to establish valid cancer diagnoses. Thus the compliance of the patient is higher than for histopathological investigations. Furthermore, an investigation of cells, as opposed to tissue, often allows an earlier diagnosis of cancer. Hence the promotion of cytopathology offers the opportunity to detect various types of cancer at early stages, thus increasing the probability of a successful therapy. Yet, the application of cytopathologic cancer diagnosis on a large scale is so far faced with two problems: the subjective microscopic evaluation of cytologic specimens is rather time-consuming, and mere morphologic inspection of cells does often not offer enough information to achieve sufficient diagnostic accuracy.

\section{Multimodal Cell Analysis}

In an interdisciplinary research project we introduced the new approach of Multimodal Cell Analysis (MMCA), which overcomes the limitations of current evaluation methods. The applied fixation of cells on microscope slides allows for repetitive staining of a smear. The set of multimodal images of the cells of interest thus produced unfolds diagnostic correlations, which are of diagnostic relevance but could not be exploited until now: Combining the specific information from different stains for each individual cell yields multivariate statistics, which lead to a considerable increase in diagnostic accuracy. The approach merges in particular a high sensitivity with a high specificity and typing accuracy. A valid diagnosis may be based on a very small number of cells.

While the basis of the diagnostic procedure is still a morphologic stain like May-Grünwald-Giemsa (MGG) or Papanicolaou (PAP), other so-called adjuvant methods are added, like DNA measurements, immunocytochemical marker demonstrations or AgNOR analyses [1][2]. So far three stains have been incorporated into the test environment (figure 1): MGG (or PAP) shows 
both nuclei and cytoplasm and only serves for inspection of morphology. The Feulgen stain (FEU) dyes DNA stoichiometrically and hence serves for ploidy measurement [3], which indicates the fraction of mitotic cells and possible aneuploidy. The silver stain (SIL) dyes less specifically, but it stains active nucleolar organising regions (AgNORs). Their number and area allows conclusions on the intensity of protein synthesis [4]. Moreover, only a combination of the results of FEU and SIL for each individual cell allows an estimation of cell proliferation on a methodological basis.

In detail the method works as follows: a specimen is fixed on a slide and is stained for a first time. Digital images of relevant cells are retrieved under the microscope, whereat their positions are stored. The slides is then removed from the microscope, and a solvent is applied in order to remove the cover glass, after which the specimen is dyed again by the next stain. Then images of the second stain are taken at the positions previously stored, and so forth. This way images from identical cells but different stains are sequentially acquired.

Due to mechanical tolerances in the gear of the microscope stage, the multimodal image sets are not sufficiently aligned for further analysis. For a precise morphologic fit the application of a coregistration algorithm is demanded. At this stage, new insights about the correlation of morphology and the respective cell physiology can already be gained by visual inspection of a cross-fading animation of corresponding images.

The following preprocessing steps, namely the segmentation of cell nuclei and a classification regarding the cell type, which may employ all previously aligned images of a multimodal set, are needed as a precondition for the subsequent evaluation of stain-specific features, such as DNA content or AgNOR features. These can finally be combined for each cell in order to gain a multivariate statistic, offering a considerable add-on to the diagnostic process, which is the method's main goal.

The overview of the whole framework of MMCA is given in [5]. The algorithms for the actual diagnostic feature extraction are currently being developed.

In this article we describe the image preprocessing that preceeds the evaluations of stains and the multivariate result output. It includes adaptive colour space transformation, automatic coregistration, segmentation, and classification of multimodal image sets. Since the preprocessing is a key factor for a clinical application of the MMCA approach, a feasibility study on a representative set of specimens was undertaken, which shall also be addressed here. Its first objective was to determine the accuracy and robustness in order to estimate the method's potential for clinical routine application. Furthermore, a supporting effect of multimodal image information to segmentation and classification was to be explored.

Below, after a short introduction to the study's design, each preprocessing step will be dealt with in the order of the given problem, the solution offered and the results. At last, results will be summarised and a future perspective will be drawn. 


\section{Experimental Design}

Seven series, each of 11 to 20 images per stain, i.e. image triples, were captured. Specimens were derived from serous effusion. Two slides contained cells from pleurisy due to chronic lymphocytic inflammation, two due to malignant pleural mesothelioma and three slides due to metastatic adenocarcinoma. Altogether 108 multimodal image triples showing a total of 693 nuclei were digitised using a Sony DXC 3-chip CCD camera mounted on a Zeiss Axioscope. Image size was $768 * 568$ pixels with an $\mathrm{x} / \mathrm{y}$ pixel resolution of 0.165 $\mu m$, which meant $127 \times 94 \mu \mathrm{m}$ on the slide. Colour depth was 24 bit. First the images of the FEU stain were captured. Their counterparts in MGG and SIL were then retrieved manually. This procedure has so far been automatised using a digitally controllable microscope.

Image processing routines were written in $\mathrm{C}++$ using the Intel ${ }^{\circledR}$ Image Processing Library (IPL) [6] and the Open Source Computer Vision Library (OpenCV) [7]. They were run on standard PC equipment.

The software was designed to run unsupervised from the first to the last preprocessing step. 


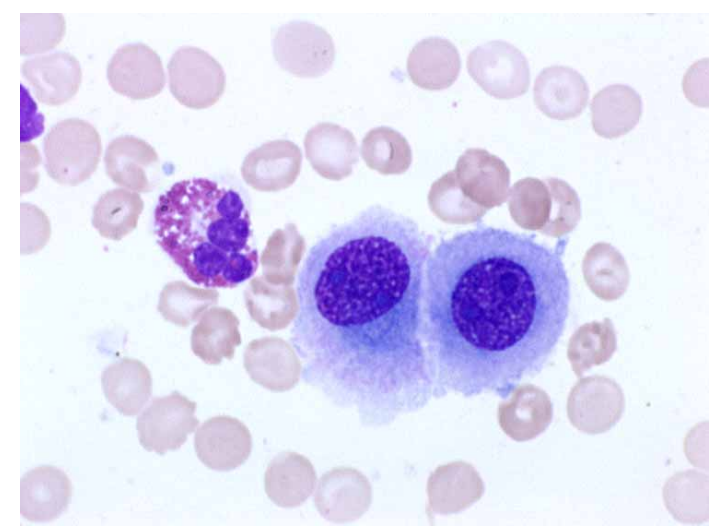

(a) May-Grünwald-Giemsa

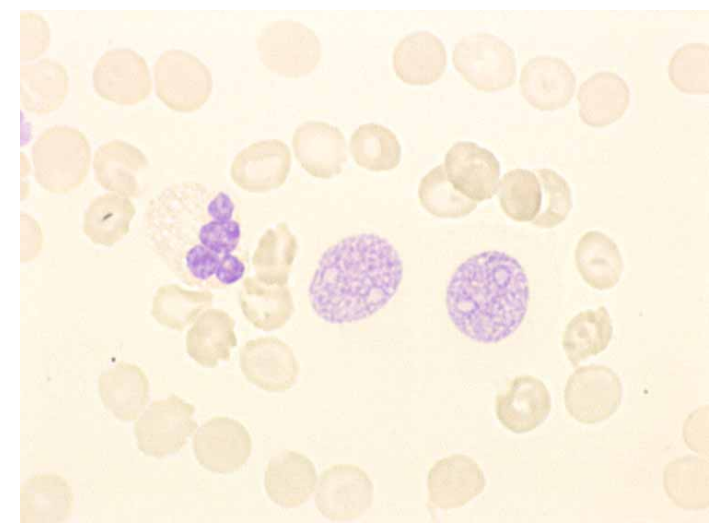

(b) Feulgen

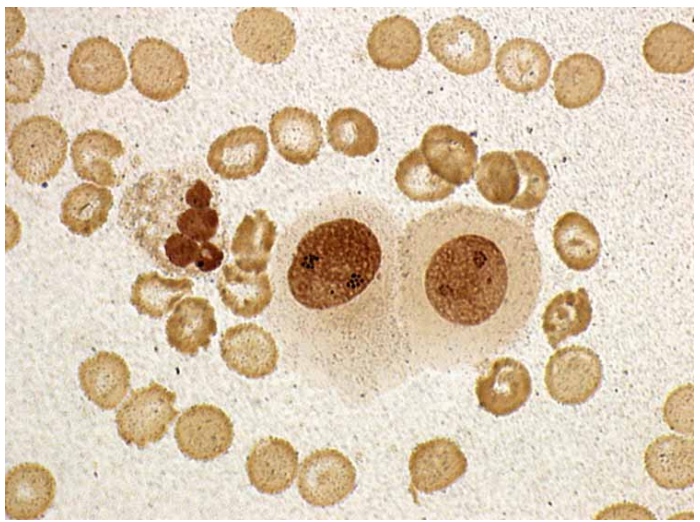

(c) Silver

Figure 1. Multimodal image set of two mesothelial cells and one neutrophilic granulocyte from a serous effusion: intensity of cytoplasm and of erythrocytes around the cells varies between the stains. 


\section{Adaptive Colour Space Transformation}

Before coregistration it is inevitable to preprocess the multimodal image triples in order to neutralise the differences in stain characteristics.

All stains show different colours but are nearly monochromatic, since information is carried merely in the staining intensity. Therefore an adaptive colour space transformation from RGB to grey scale is appropriate.

\subsection{General Approach}

Each stain is treated individually. For each series an accumulated RGB histogram of all images is built. Then a cluster analysis is performed on the histogram returning a foreground and background colour distribution. These are passed on to the Fisher discriminant algorithm [8], which provides for a transformation to grey scale with maximal contrast between two colour populations, given a mean and a covariance matrix each. This way colour space transformation is adaptive to differences in individual staining results or image capture settings, which may vary between the image series. The only fixed parameters are the start values of the distributions for cluster analysis, which are robust, because of the small number of distributions and the well-known characteristics of the stains.

\subsection{Results}

As the algorithm is robust, its efficiency regarding the grey level contrast achieved should be compared with standard transformation methods. For this purpose the contrast between nuclei and background was measured because it is the most important feature for segmentation. Anticipating the result of the segmentation step, contours found by the snake method could serve as reference contours. The average underlying image edge strength of a contour was evaluated using the values of the derivated Gaussian of its normals (see 5.2), returning negative values for the nucleus-to-background contrast as can be seen in table 1 for the example of the Feulgen stain. Results of the Fisher discriminant were compared with other methods such as mean RGB value or channel extraction from both RGB and HSV models. The minimal, average and maximal values refer to whole image series.

The result of the Fisher discriminant surpasses most other methods by far; only hue channel extraction shows similar contrast. However, the adaptivity of the combination of of histogram clustering and Fisher discriminant ensures constantly high contrasts and compensates for intra-stain differences due to variable staining conditions. 
Table 1

Comparison of Colour Space Transformation Methods (Feulgen stain)

\begin{tabular}{|c||r|rrr|}
\hline Transformation Method & $\begin{array}{r}\text { Average } \\
\text { Contrast }\end{array}$ & $\begin{array}{l}\text { Enhancement by Fisher Discriminant [\%] } \\
\text { min }\end{array}$ & \multicolumn{1}{|c|}{ max } \\
\hline \hline$\frac{1}{3}$ (red + green + blue) & -19.1 & 23 & 38 & 62 \\
\hline red channel & -4.7 & 365 & 457 & 639 \\
\hline green channel & -23.6 & 00 & 60 & 30 \\
\hline blue channel & -16.5 & 38 & 2 & 101 \\
\hline hue & -25.8 & -8 & 255 & 15 \\
\hline saturation & -7.4 & 122 & 101 & 634 \\
\hline value & -13.1 & 76 & 0 & 139 \\
\hline Fisher discriminant & -26.4 & 0 & & 0 \\
\hline
\end{tabular}

\section{Coregistration}

If only mechanically retrieved, the images are not sufficiently aligned to allow further multimodal processing. While the nuclei have diameters from about 10 to $20 \mu \mathrm{m}$, other structures of interest lie in the order of $1 \mu \mathrm{m}$ (e.g. AgNORs). Therefore an alignment of subpixel accuracy should be reached. Initial misalignment was up to $30 \mu \mathrm{m}$ of translation and up to $3^{\circ}$ of rotation.

\subsection{General Approach}

Alignment is a precondition to multimodal segmentation. Segmentation is necessary to understand the image scene (e.g. positions of the cell nuclei). Consequently the bare images have to be taken as a whole for the alignment process. A common method for coregistering two images [9] is to construct a function $F$ from the following two components: one image is chosen as the reference image $R$ (here: FEU), the other as the object image $O$ (MGG resp. SIL), which is passed through an affine transformation $T(p, O)$ with parameter vector $p$ in the first step. Second the reference image and the transformed object image are compared by means of a real function $C$ called the cost function that measures their dissimilarity. It should be sensitive to misalignments while insensitive to other differences. Here a scaled variant of the pixelwise L2 norm is utilised. Be $R(x, y)$ and $O(x, y)$ pixel intensities of an $X \times Y$ sized reference resp. object image, the cost function is

$$
C(R, O)=\frac{\frac{1}{X Y} \sum_{x, y}(R(x, y)-O(x, y))^{2}}{\frac{1}{X Y} \sum_{x, y}(O(x, y))^{2}}
$$


The overall function is

$$
F(p)=C(R, T(p, O))
$$

where $T(p, O)$ is the transformed object image and $\mathrm{T}$, in our case, a $2 \mathrm{D}$ rigid body transformation. $F$ is minimised in order to obtain the parameter set $p$ resulting in the smallest dissimilarity between reference and transformed object. Interpolation is bilinear. $F$ is minimised using the Powell algorithm [10].

\subsection{Avoiding Local Minima}

This method is wide-spread in fMRI-realignment applications [9], i.e. the realignment of brain images, which contain only one large object. In contrast to that task, here an image contains several small objects, the nuclei, and large areas of uniform background. The function $F$ of two brain images looks more or less like a single parabola, while here it is a constant, interrupted by local minima where any pair of objects overlap. The global minimum is reached at the overlap of corresponding objects. Local minima are a major obstacle to numerical function minimisation.

The problem is solved by means of a Gaussian pyramid [11], a multi-resolution technique. The resolution of the images is repeatedly decimated by a factor of two in order to reduce the level of detail and bring distant structures closer together. The effect on the cost function can be seen in figure 2. The global minimum of the cost function in 2(b) is surrounded by local ones. As indicated by the arrows, they originate from the overlap of non-corresponding nuclei. These local minima nearly vanish on the 6 th level of the pyramid $(2(\mathrm{c}))$. At this level the algorithm starts and passes the resulting parameters down to the next more detailed level to start with. This procedure is repeated down to the original image level.

Though converted into grey scale, the characteristics of the different stains still differ: the Feulgen stain dyes only nuclei (cf. fig. 1) while MGG also displays cytoplasm and, with less contrast, erythrocytes. SIL is least specific and dyes both erythrocytes and cytoplasm nearly as intensely as nuclei. It is necessary to roughly remove those structures in order to align MGG and SIL images with FEU images. Otherwise the probability of local minima is unnecessarily high, e.g. because of an overlap of nuclei and erythrocytes. besides, $F$ becomes less sensitive to changes of alignment, which leads to a broader global minimum (see figure 3(a)). To remove them, a pre-segmentation of nuclei in the FEU images is performed using the cluster analysis of the RGB histogram from 3. The resulting foreground colour distribution is selected in order to determine the sum of corresponding image pixels. To all other images of the triple a 


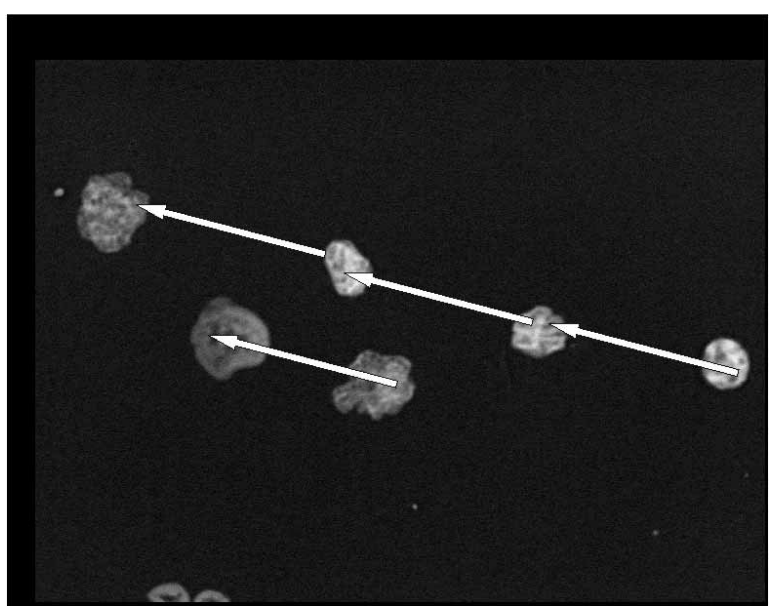

(a)

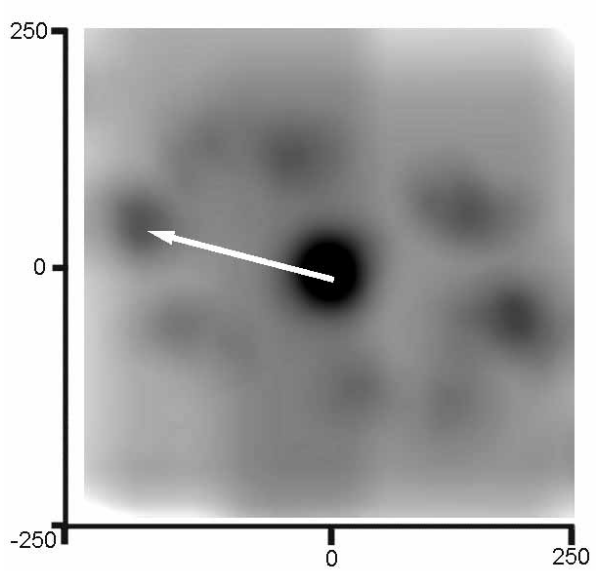

(b)



(c)

Figure 2. Avoidance of local minima of a cost function using a Gaussian pyramid: 2(a) FEU image in grey-scale, 2(b) cost function of 2(a) and the corresponding SIL image showing translations of \pm 250 pixels, 2(c) same cost function, but at the 6 th level of a Gaussian pyramid.

thresholded is applied in order to adapt their numbers of nonzero pixels to the latter. Coregistration is performed on the processed images. The improvement of the cost function can be seen in figure 3 .

\subsection{Results}

The results were evaluated visually using RGB overlay images of all three stains. Figure 4 shows an example image before and after coregistration. The whitish appearance of nuclei without any coloured margins in the final image demonstrates the correctness of coregistration. For each image triple two coregistrations were needed (FEU-MGG and FEU-SIL). In total 215 of 216 


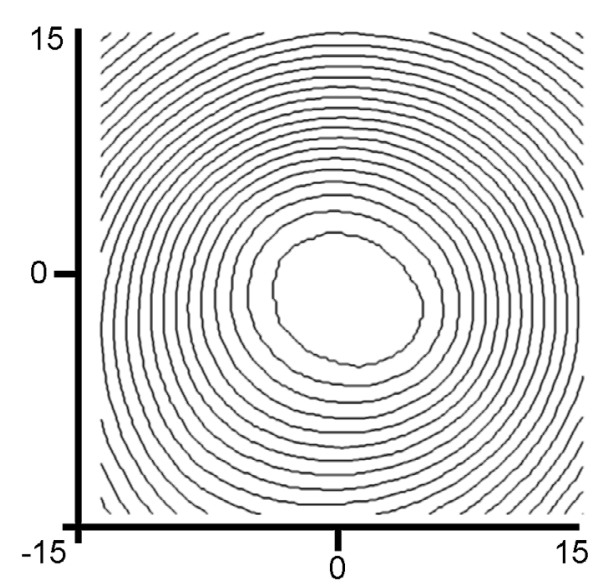

(a)

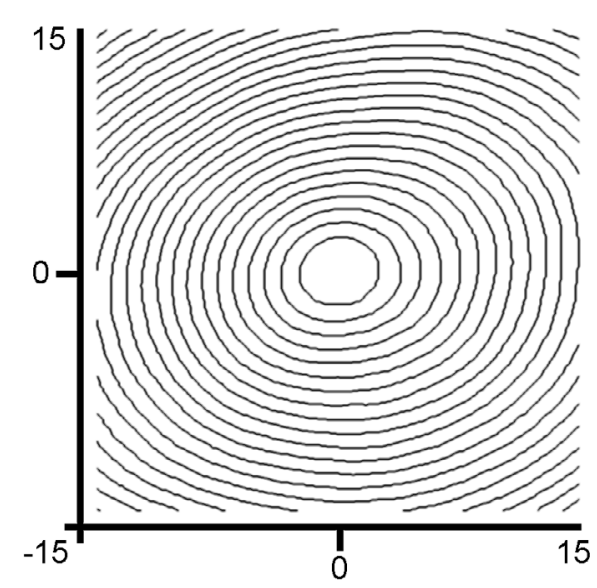

(b)

Figure 3. Refining the sensitivity of a cost function: 3(a) FEU-SIL cost function within translations of \pm 15 pixels around the global minimum, illustrated with contour lines, 3(b) Cost function of the same images, but after foreground pixel equalisation.

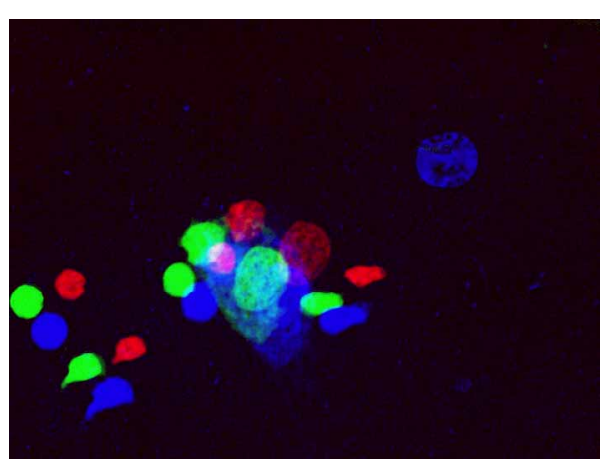

(a)

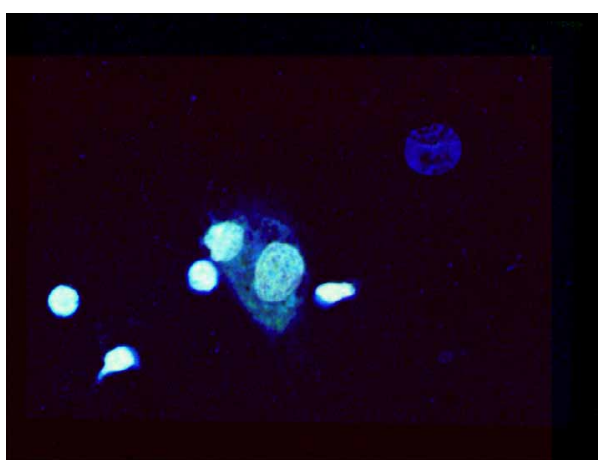

(b)

Figure 4. Overlay visualisation of an image triple: FEU red, MGG green, SIL blue, 4(a) before coregistration, 4(b) after coregistration.

coregistrations succeeded, allowing multimodal processing of 107 of the 108 images triples (99.1\%). The cause of the failure was an SIL image with nuclei hardly standing out of the cytoplasm. In this condition, the global minimum of the cost function is blurred.

\section{Segmentation}

Automatic segmentation of cell nuclei is the most demanding step of the preprocessing cascade. Manual corrections are tedious and are therefore out of the question in large scale clinical use. Prior works in this area mostly made use of 
threshold based or morphologic segmentation methods such as the watershed algorithm. But these do not take object shape into consideration, so retrieved contours tend to have irregular notches. Furthermore, automatic separation of touching nuclei is not facilitated.

Active Contours result in smooth, plausible outlines, but, according to their reputation, at the cost of high dependency on initialisation, lack of adaptiveness, and finally imprecise segmentation results, especially of concave objects. This shall be disproved.

\subsection{General Approach}

A contour-based approach was preferred in contrast to region-based segmentation. Cubic B-splines (CBS) [12] were chosen as an object representation model for segmentation. Both description and modification of closed curves can be handled with CBS fairly easy. This holds also for other parameters like the first and second derivative of the contour, which are needed to evaluate object features for classification (cf. section 6). This advantage is lost when objects have sharp notches, but this is not the case for smooth membranes like those of cell nuclei. A segmentation method that lets a contour model iteratively converge toward an underlying image feature is called active contour or snake [13]. Movement is driven by external image forces, usually derived from image edges, and internal forces that model features like stiffness or tensibility. Because of these simulated physical features the method is known to be robust against noisy or partially missing image information.

Forces act on and between points distributed along the snake, which are here called knots. Both their number and the fraction of image forces versus the inner forces make for the degree of freedom of the snake movements. They need to be balanced in order to make the contour model both flexible enough to enclose the image contour and stiff enough not to be partially distracted by other image structures, which is not trivial, particularly if the variance in object appearance is high, as with cell nuclei.

These characteristics of a snake may also be intrinsic to the contour model: When using B-Snakes [14][15], based on B-splines [16], the stiffness of the contour is solely induced by the dependency of the knot positions due to the spline polynomials, rendering explicit inner forces unnecessary. Stiffness is modeled by the density of knots and the number of sampling points between them. The latter assure a fine sampling of the image; they influence movements of their neighbouring knots but do not increase the degree of freedom of contour movements. This method results in a much smaller number of knots and reduction in computational cost. Furthermore, the adjustment of the stiffness parameter becomes more intuitive (cf. 5.4).

In every iteration the snake is moved within a defined neighbourhood driven by the image forces or in other words, reducing its potential energy. Evaluat- 
ing new positions for the $k$ knots of a snake during an iteration can be seen as a $k$-dimensional function minimisation. In this work a sufficiently robust greedy algorithm is utilised, which moves each knot along its contour normal, here called the scan line.

External forces of different kinds, each applied on an arbitrary modality, can easily be incorporated into the snake model. Here they are summed up using weight factors.

\subsection{Image Forces}

The most common kind of image forces for snakes are derived from image edges.

These are usually implemented by means of common image edge filters that assign high pixel values to edges. The thereby induced loss of information on the edge direction disables snakes to differentiate between neighbouring objects (figure 5(b)), a major cause to the regularly criticised high dependency of snakes on initialisation. In this work contour normals are convolved by a derivated Gaussian, preserving the edge direction and thus such differentiation (figure 5(c)). This way also the expected edge direction is optimally taken into account at each iteration of the snake. As a side-effect, image normals can be made longer without the risk of attraction by other objects, facilitating the segmentation of concave objects (fig. 6(a)). The filtering must be recomputed every time a knot is moved. However, it consists in a fast $1 \mathrm{D}$ convolution.

A drawback of using solely edge forces is that only information on image discontinuity is used, not on continuity, i.e. region information. Therefore, grey scale values themselves were added for the discrimination between several kinds of edges, such as

(1) edges of structures within a nucleus,

(2) nucleus-to-cytoplasm or nucleus-to-background edges,

(3) cytoplasm-to-background edges,

(4) background-to-background edges induced by artefacts.

Nucleus segmentation should obviously only be sensitive to the second kind of edges. 


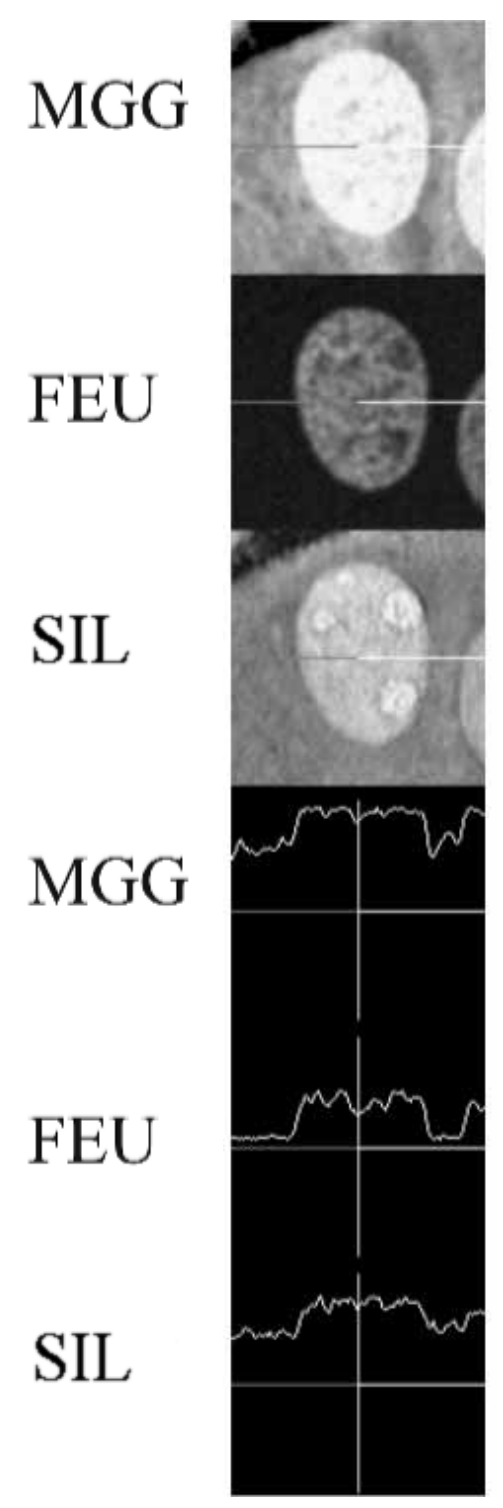

(a)

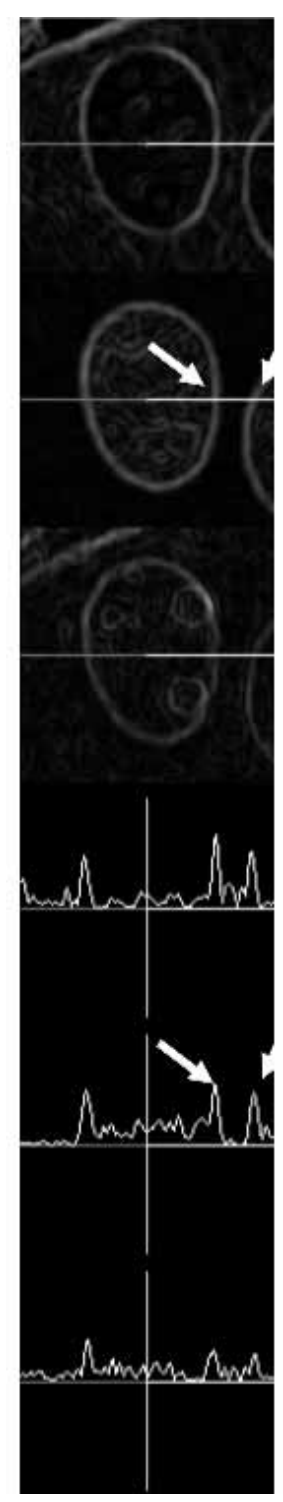

(b)

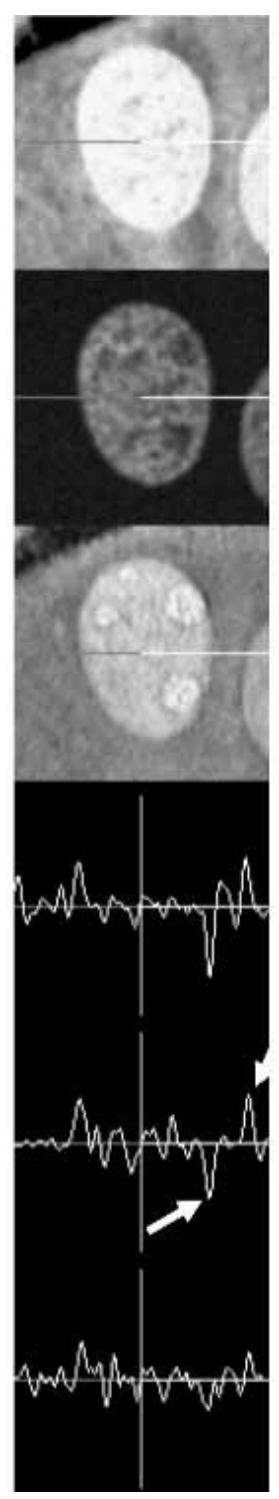

(c)

Figure 5. Scan lines and the impact of directed edge filtering: 5(a) multimodal profiles of a nucleus using the scan line model (rear grey, front white) and the identity function, 5(b) conventional edge profile as identity function on an edge-filtered image; all edges are maxima, 5(c) edge filtering along the scan discriminates between foreground-background edges (minima) and background-foreground edges (maxima).

\subsection{Initialisation}

A major drawback of snakes is their dependency on initialisation. At this step the result of the adaptive colour space transformation (3) can be used once 


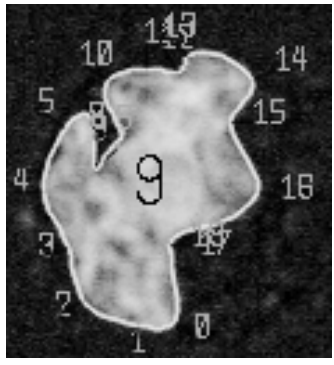

(a)

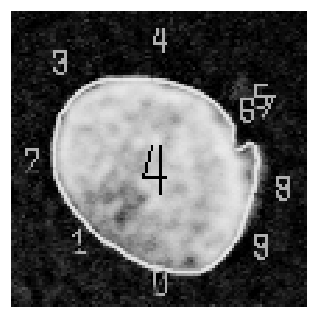

(b)

Figure 6. Two examples of B-Snakes and variable knot density: 6(a) irregular nucleus, 6(b) only ten knots describe the contour, with a higher density around the notch.

more (cf. 4.2). Let $F G_{i}$ be the set of foreground pixels from stain $i$. A minimal set of foreground pixels is extracted combining all stains:

$$
F G=\bigcap_{i} F G_{i}
$$

This pre-segmentation method returns good initial estimates for the nuclei while in the resulting binary image residues of other structures are broken up into fragments that can easily be rejected according to their smaller sizes.

Next for each binary object of nucleus size a contour is extracted using a morphological gradient (here: dilation and subsequent subtraction of the input image). The snake is initialised on this binary contour (fig. 7).

\subsection{Knot Insertion}

On the one hand, the more complex a contour is, the more knots in the spline are needed to approximate it. On the other hand, the more knots there are along a spline, the more independent become the segments between them on the global shape and the more likely they are attracted by other image features than those of the nucleus contour. Furthermore, complexity may vary along a contour. For example, a contour may be fairly smooth on most of its outline and have only one notch. Considering these facts, a variable density of knots along the snake is desirable (see fig. 6(b)). In our implementation the contour model is initialised with only four knots. Every time the snake has converged (i.e. the maximal knot movement has under-run a certain threshold), the segments between knots are tested for a distance criterion according to the image feature, and knots are inserted only where necessary using the Böhm algorithm [12]. The average or maximal squared distance proved useful, as it resulted in accurate segmentations while retaining the snake noise-resistant. In case knots were inserted, the snake is started again and so forth (cf. figure 7). 


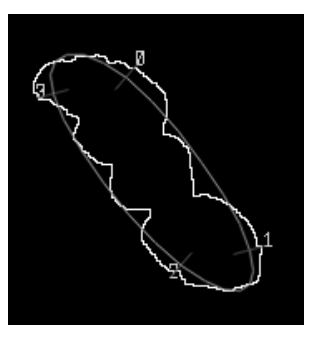

(a)

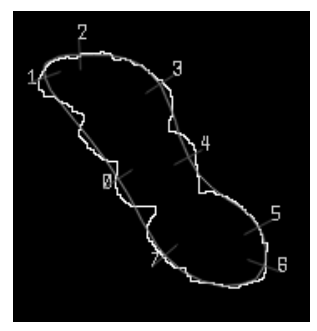

(b)

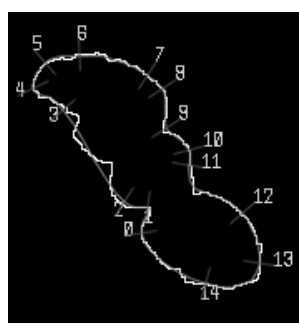

(c)

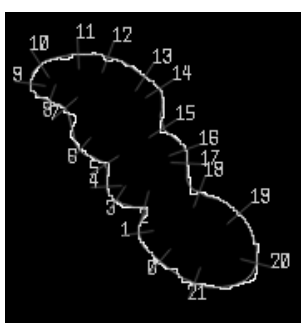

(d)

Figure 7. Knot insertion: 7(a) convergence with four knots after 54 time steps, 7(b) convergence with eight knots, $t=119,7(\mathrm{c})$ convergence with 15 knots, $t=172,7(d)$ termination with 22 knots after 214 time steps.

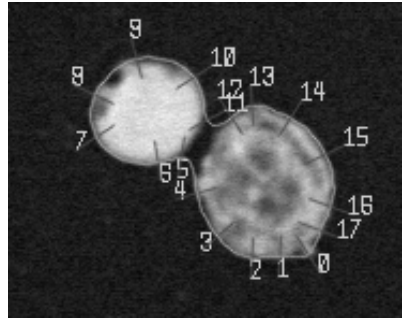

(a)

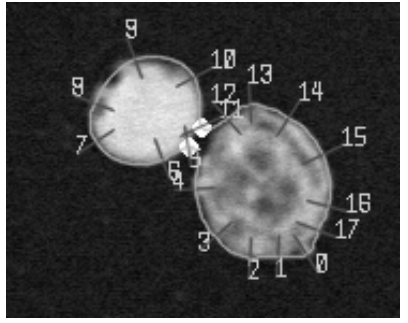

(b)

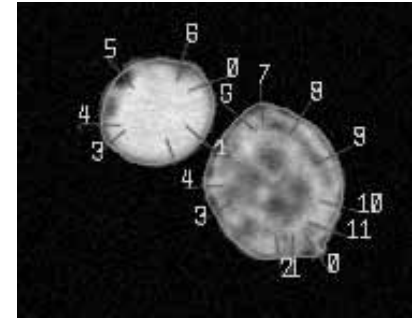

(c)

Figure 8. Split of a snake due to distance criterion: $8(\mathrm{a}) \mathrm{t}=0,8(\mathrm{~b})$ detection and split at $\mathrm{t}=29,8(\mathrm{c})$ both segmented nuclei.

\subsection{Automatic Separation}

After the initialisation the snake is superimposed on the grey scale image triple. Forces can be defined for each image and be combined so that the resulting force is a weighted sum of arbitrary forces from all images (cf. 5.2). The segmentation algorithm is basically the same as during the initialisation. However, the snake occasionally encloses two or more nuclei after initialisation. This problem is solved by two approaches. First, if the nuclei are sufficiently far apart, the snake will move into their interspace from one or two sides once it is superimposed on the grey scale images, so it can be automatically separated under the condition that the distance of two sampling points is

(1) below a certain level, according to their Euclidian distance, and

(2) above a second level, according to an arc length measure (e.g. relative knot distance) (cf. figure 8).

Second, if there is hardly any space between the nuclei, the snake will have two opposite concavities after convergence, assuming that nuclei are approximately circular and of similar size. The contour is then checked for concavities using its derivatives. For each pair of them the following two features are evaluated: 


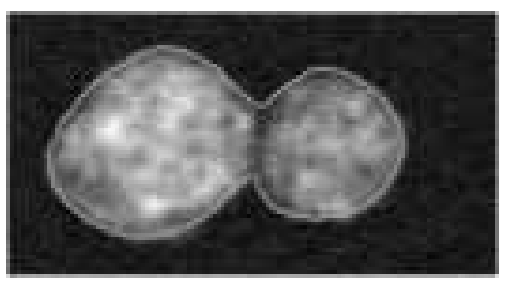

(a)

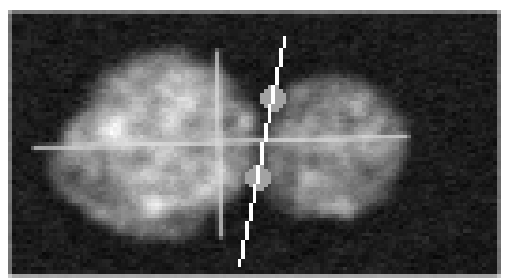

(c)



(b)

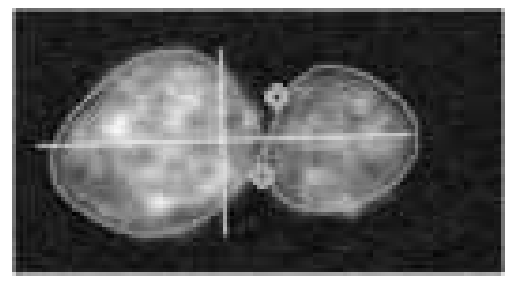

(d)

Figure 9. Split of a snake due to concavity criterion: 9(a) contour after segmentation, 9(b) detected concavities, 9(c) principal axes and line connecting concavities; the angle between the line and the principal axis is near $90^{\circ}$, the linear path between concavities is relatively dark, 9(d) split contours, ready for individual segmentation.

(1) The direction of the connecting line in relation to the principal axis of inertia of the whole object: if approximately perpendicular, the line may separate a system of two objects (cf. figure 9). The principal axis is determined using central moments of a region representation of the object.

(2) The mean grey level along the line normalised by the mean grey level of the whole object. A dark path indicates background or marginal areas.

The decision whether to split the object or not is based on these features.

\subsection{Results}

As expected, FEU came out as the most suitable stain for segmentation, since it does not dye cytoplasm or cell types containing no DNA. FEU produced the most consistent contrasts across the image series, too. This, on the other hand, leads to a drawback of FEU: DNA is stoichiometrically dyed, so the chromatin patterns, i.e. a texture of the nucleus due to the state and structure of the DNA, produces superior edges inside the boundary of the nucleus. This can make a snake based on edge forces break into the nucleus.

Segmentation errors due to this effect could be reduced by a further modality: a combination of FEU and MGG edge forces at a ratio of 7:3 since MGG dyes the inner parts of nuclei more homogeneously. The additional force opened, however, a new source of errors: where nuclei were surrounded by a rather thin layer of cytoplasm, the snake occasionally broke out towards the cytoplasmto-background edge, thus partially reversing the gain. 




(a)
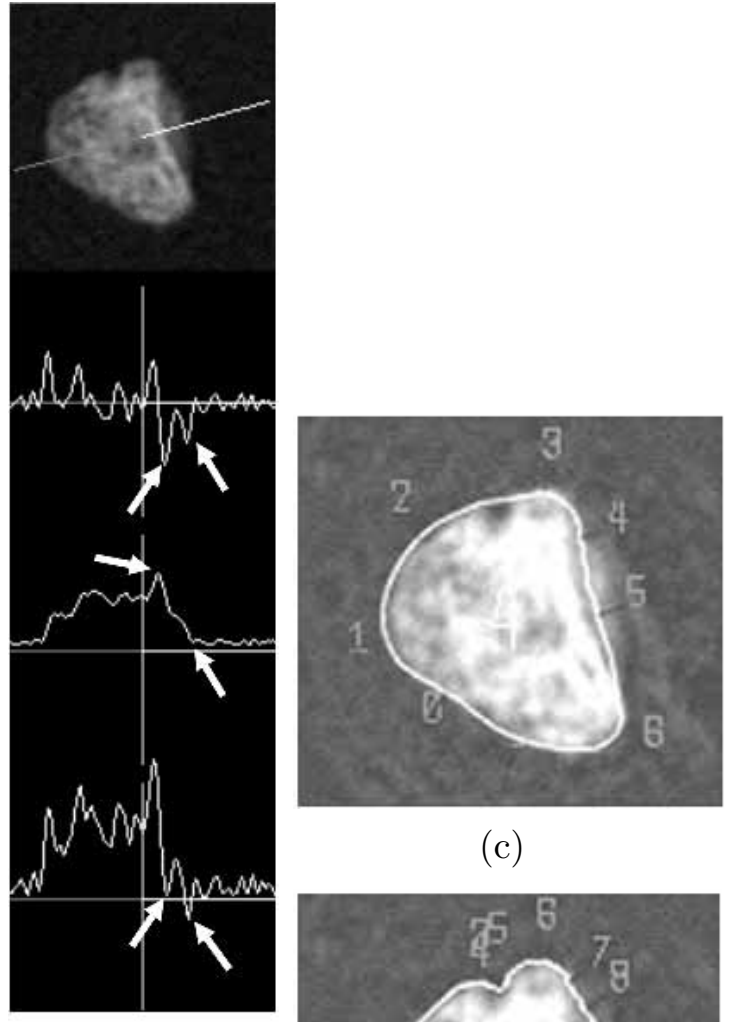

(c)

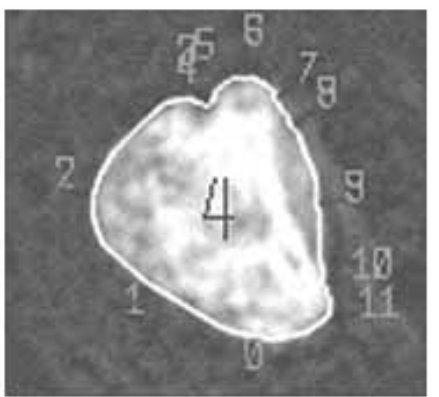

(d)

Figure 10. Image forces and their impact on segmentation: 10(a) combination of edge forces from FEU and MGG, 10(b) same object but with a combined edge-to-identity force; the inner edge value is increased by the underlying image intensity information resulting in a new global minimum at the outer edge, 10(c) segmentation according to forces from 10(a); although the MGG fraction weakens the effect, the snake breaks into the object because of high-contrast inner structures, 10(d) segmentation according to 10(b); the nucleus is segmented correctly.

Finally the approach presented in section 5.2 improved the segmentation accuracy substantially: MGG edge information was replaced by the FEU identity function in the force set, i.e. the grey values of FEU were added at an edgeto-identity ratio of $4: 1$. The lower grey value level of nucleus-to-background edges compared to those of chromatin patterns resulted in an tremendous stabilisation when using the identity function (see figure 10).

Also the exclusion of artefacts and lysed cells by means of size and intensity criteria worked sufficiently well. 


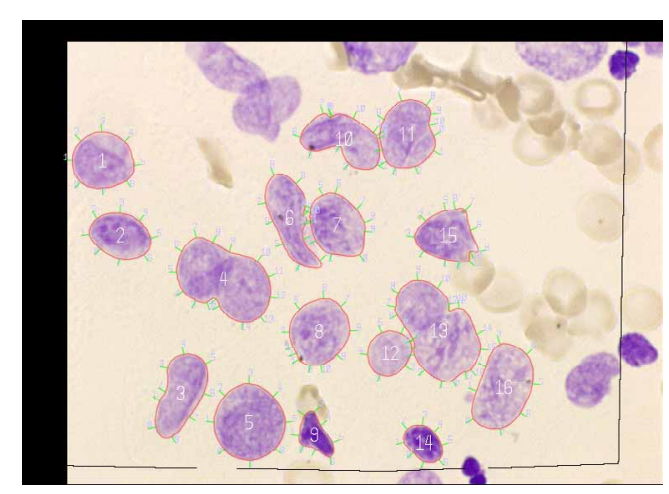

(a)

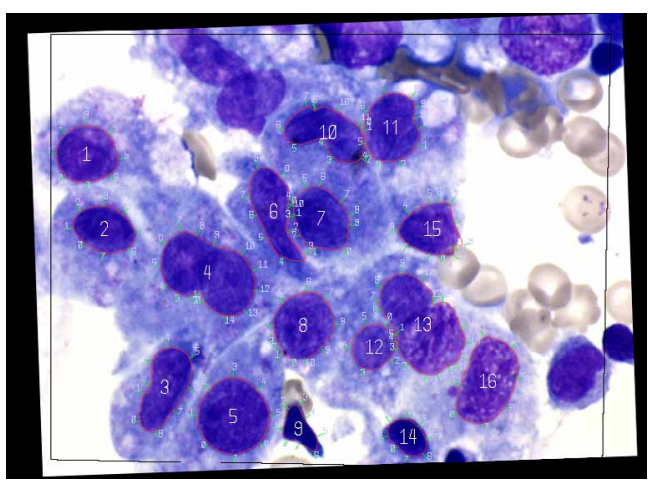

(b)

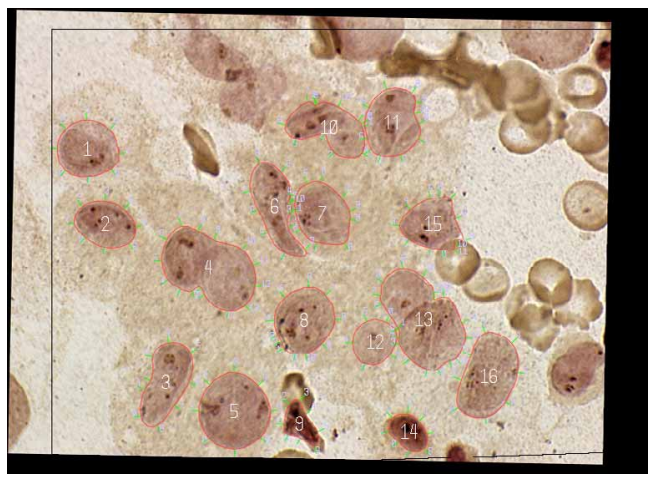

(c)

Figure 11. Image triple with overlayed segmentation: 11(b) FEU, 11(a) MGG, 11(c)SIL. All nuclei resp. clusters of overlapping nuclei are correctly segmented using FEU, despite partially strong inner edges and some nuclei nearly touching each other. Only objects within the common area (black frame) were processed. Very slight aberrations between segmentations and real contours can be found in MGG and SIL. Such changes result from shrinkage of proteins due to secondary formalin fixation.

Automatic separation of pairs of pre-segmented nuclei further reduced the remaining amount of segmentation errors. The overall success rate yielded $92.6 \%$ (642 of 693 nuclei). An image triple with overlaid segmentations is shown in figure 11. 


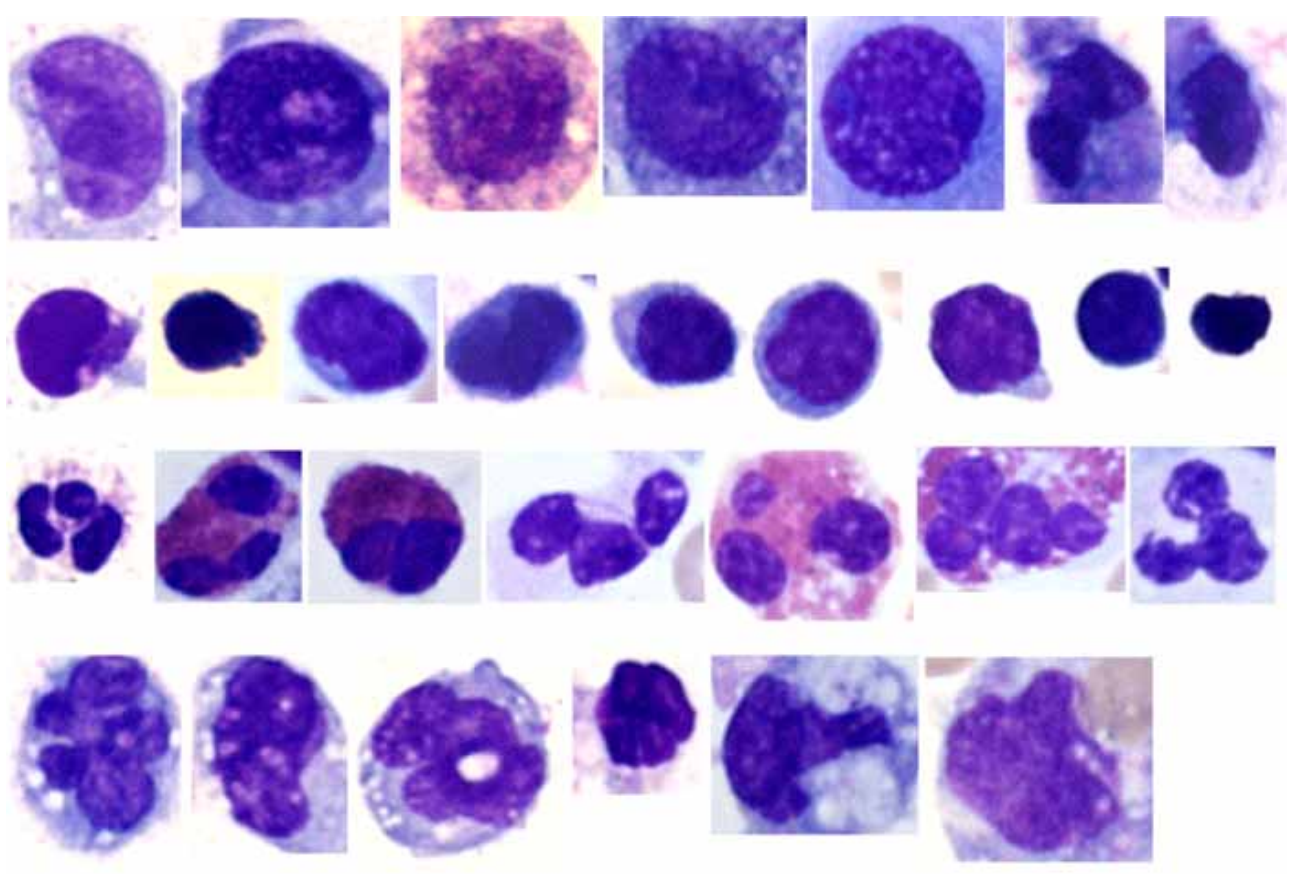

Figure 12. MGG-stained examples of the four classes, top down: mesothelial cells, lymphocytes, granulocytes, macrophages.

\section{Classification}

For the automatic analysis the nuclei need to be classified into cells to be measured and reference cells, which are used for calibration. This leads to a classification regarding to all occuring cell types, which are in case of serous effusions: mesothelial cells (ME), lymphocytes (LY), granulocytes (GR), and macrophages (MA), see figure 12. They differ in various aspects such as size, form, and texture. At this point the concept of multimodality offers a striking advantage since the correlation of different stains delivers valuable features for the classification.

The similar appearance of ME and MA nuclei is a challenge in this task, making the discrimination difficult even for an instructed non-specialist. In addition, the amount of available training data in this first study was very small and classes were not uniformly distributed: $384 \mathrm{ME}, 176 \mathrm{LY}, 38 \mathrm{GR}$, and 44 MA nuclei.

\subsection{General Approach}

In order to prove the feasibility of classification based on the automated segmentation, a basic set of eight features was extracted: area, perimeter, mean grey value, standard deviation of grey value, circularity, eccentricity, bending 
energy, and fraction of surrounding cytoplasm. Seven features were extracted from FEU, while the last one indicating by what fraction around its perimeter a cell nucleus is surrounded by cytoplasm was retrieved from MGG.

Two different classification approaches were implemented: a Bayesian classifier based on Gaussian mixture densities and a kNN-classifier [17].

\subsection{Results}

Due to the small amount of data it was impossible to first generate an optimal parameter set on a defined training set and to measure a valid recognition rate using a disjoint data set. Therefore results were determined for each of the classifiers' parameter combinations, e.g. one, two, or four mixtures within a mixture density for the Bayesian classification or different k-values for the $\mathrm{kNN}$ classifier.

For each parameter set the recognition rate was evaluated by means of the leaving-one-out method [17]: one image series was used for classification, while the other provided the training data. This step was performed for all permutations and the resulting recognition rates were averaged.

The optimal recognition rate of the Bayesian classifier was $86.1 \%$, the rate of kNN $87.5 \%$. While results of the Bayesian classification showed significant differences for different parameter settings, $\mathrm{kNN}$ classification changed only marginally when increasing or decreasing the k-value. Therefore the result of the latter can serve as a quality measure despite the restrictions mentioned above.

\section{Overall Results}

The preprocessing for Multimodal Cell Analysis is a multi-level procedure. Thus its overall efficiency must be derived from the single levels, but taking into account also the mutual dependencies of their outcomes. Most probably, for example, misalignments of about $1 \mu \mathrm{m}$ after the coregistration do not impact on the further steps, while imprecise segmentations may adulterate the results of subsequent classification and, of course, diagnostic feature evaluation.

Additionally the reversibility of errors at the different levels should be factored into a quality measure: The results of the preprocessing procedure must be inspected visually by the pathologist in advance of the calculation of diagnostic parameters. This allows for the manual correction of some errors. Toggling of false classification results is acceptable, whereas manual coregistration of misaligned images or segmentation of incorrectly segmented nuclei is not because it would simply take too long. 
Hence a method of deriving "the true" overall efficiency cannot be clearly defined. Nevertheless a final quality measure shall be given. The success rates of the individual processing steps are

- coregistration $99.1 \%$,

- segmentation $92.6 \%$,

- classification $87.5 \%$.

Their product, $80.3 \%$, may be regarded as a "worst-case"' estimation for the overall efficiency.

\section{Summary and Perspective}

The first objective of the described work was to develop a fully automatic preprocessing algorithm for MMCA and to evaluate its appropriateness for clinical routine application. It could be stated that the results fulfilled the given requirements. As an additional option, "'difficult"' nuclei could be automatically rejected by means of uncertainty measures, as is customary in existing applications. This would further improve the success rate.

The second objective was to find out whether the employment of multimodal information could support the steps of segmentation and classification. As for classification this is the case because object features were extracted from different stains. So far, segmentation results were best when using only the FEU stain. Improvements through multimodality will, however, be further investigated. A first point will be the choice of image forces from different stains. Furthermore, the snake topology will need to be optimally adjusted to the multimodal concept. So far inspections of the segmented nuclei showed tiny irregular differences in size between the same nuclei in different stains (cf. figure 11), which result from shrinkage of proteins due to secondary formalin fixation. Improvements of the precision of the segmentation are expected if such systematic deviations of the contour of the same nucleus in successive stains will be incorporated into the snake model.

Concerning to the preprocessing steps addressed here, there are two challenges for the near future. First, in the specimens tested the distance of nuclei was mostly large enough to avoid interference during segmentation. The system should, however, also be able to handle specimens with cell clusters, i.e. with many proximate or overlapping nuclei. So far only pairs of nuclei can be separated, and overlap is not detected.

As a second challenge, the recognition rate in cell type classification has to be improved. More features will be added and further research on the classifier will be performed. So far a recognition rate of $87.5 \%$ could be reached despite the small number of cells at hand in the study. This number is being raised from the presently investigated 642 cells to approx. 90,000 by an ongoing med- 
ical field study for diagnostic evaluation of the MMCA method.

For this purpose, a prototype of the described approach has been implemented in a cytopathology workstation, which includes a graphical user interface and a database for images and related data. The automatic evaluation of diagnostic features (DNA analysis, AgNOR count) is to be developed and will be evaluated on the results of the mentioned field study.

\section{References}

[1] BöCKIng, A.: Towards a Single-Cell Cancer Diagnostics, The distinguished Ploem Lecture. In 7th congress of the European Society for Analytical Cellular Pathology, Caen, France, April 2001.

[2] Böcking, A.: Zytopathologie. In Thomas, C. (editor): Histopathologie, chapter 16, pages 411-440. Schattauer, Stuttgart, New York, 1998.

[3] Böcking, A.: DNA Measurements - When and Why? In WiED GL, Keebler CM, Rosenthal DL (editor): Compendium on Quality Assurance, Proficiency Testing and Workload Limitations in Clinical Cytology, Tutorials of Cytology, Chicago, 1995.

[4] RÜschoff, Josef:

Nukleolus Organisierende Regionen in der Pathomorphologischen Tumordiagnostik, volume 139 of Veröffentlichungen aus der Pathologie. Gustav Fischer Verlag, 1st edition, 1992.

[5] Meyer-Ebrecht, D., A. Böcking, J. Stockhausen and T. Würflinger: Multimodal Cell Analysis: Digital Image Processing Enhances Cytologic Cancer Diagnoses. In submitted to IEEE Mag EMB.

[6] Inte ${ }^{\circledR}$ Image Processing Library. http://www.intel.com/support/performancetools/libraries/ipl/index.htm.

[7] Open Source Computer Vision Library. http://www.intel.com/research/mrl/research/opencv/.

[8] Fisher, R. A.: The use of multiple measurements in taxonomic problems. Annals of Eugenics, 7(2):179-188, 1936.

[9] Woods, R.P., S.T. Grafton, C.J. Holmes, S.R. Cherry and J.C. MAZZIOTTA: automated image registration: I. General methods and intrasubject, intramodality validation. Journal of Computer Assisted Tomography, 22:141154, 1998.

[10] Press, W. H., S. A. Teukolsky, W. T. Vetterling and B. P. Flannery: Numerical Recipes. Cambridge University Press, Cambridge, 2nd edition, 1992.

[11] JÄHne, Bernd: Digital Image Processing: Concepts, Algorithms, and Scientific Applications. Springer-Verlag, Berlin, Germany, 2nd edition, 1993. 
[12] Foley, J., A. van Dam, S. Feiner and J. Hughes: Computer Graphics Principles and Practice. Addison-Wesley, Reading, Mass., 1990.

[13] Kass, M., A. Witkin and D. Terzopoulos: Snakes: Active Contour Models. In Proc. of IEEE Conference on Computer Vision, pages 259-268, London, England, 8-11 1987.

[14] Menet, S., P. Saint-Marc and G. Medioni:

B-snakes: implementation and application to stereo. In Proceedings of Image Understanding Workshop, 1990.

[15] Brigger, P., J. Hoeg and M. Unser: B-Spline Snakes: A Flexible Tool for Parametric Contour Detection. IP, 9(9):1484-1496, September 2000.

[16] Sonka, M., V. Hlavac and R. Boyle: Image Processing: Analysis and Machine Vision. Chapman And Hall Computing, London, 2nd edition, 1995.

[17] Duda, Richard and Peter Hart: Pattern Recognition and Scene Analysis. John Wiley and Sons, 1973.

Thomas Würflinger, MSc, studied informatics at Aachen University, where he received his Diploma in Informatics in 2002. He was awarded the Springorum Memorial Medal for his outstanding achievements. He has been member of the MMCA research team since 2001 and he is currently pursuing his $\mathrm{PhD}$ research in the Faculty of Electrical Engineering and Information Technology. His research interests are focussed on medical image processing and pattern recognition.

Jens Stockhausen received his Diploma in Solid State Physics from the II Pysical Department of Aachen University in 1999. Since then he is working as $\mathrm{PhD}$ fellow at the Institute for Measurement Technique and Image Processing, Aachen University. Besides the development of algorithms for the analysis of light-optical microscopic images he is in charge of the application environment of MMCA.

Dietrich Meyer-Ebrecht, Univ-Professor Dr-Ing, studied EE at Technische Universität Carolo Wilhelmina of Braunschweig from 1958 to 1964, where he got his Diploma EE in 1964 and his PhD in 1974. In 1964 he started his professional career as a research fellow at Philips Research Labs, Hamburg, where he was in charge of several research groups from 1969 to 1983. In 1984 he was appointed Full Professor and Director of the Institute for Measurement Techniques and Image Processing of Aachen University. Since 1996 he is Chairman of the Board of Forum Informatik of Aachen University. His research is devoted to vision systems, image analysis and human interaction with a focus on medical applications. He was involved in various interdisciplinary research projects on diagnostic image analysis, surgery navigation, PACS, and hospital 
information systems. He has published more than one hundred scientific papers. For pioneering work in medical image analysis and user interface design he got the DAGM award in 1993 and the GfR award in 1992. He holds 31 patents and has submitted 8 patent applications.

Alfred Böcking, Cytopathologist, Univ-Professor Dr med, is a graduate of the University of Freiburg, Germany where he received his MD in 1971 and his PhD in 1980. In 1982 he was appointed Head of the Department of Pathology and Cytology of the Aachen University. In 1994, he was appointed to his current position of Director of the Institute of Cytopathology, University of Düsseldorf. He is author of some 200 publications in scientific journals and books and co-inventor of the multi-modal cell analysis. He has done pioneering work in the development of systems for diagnostic DNA image cytometry. In recognition of his work he was awarded the Gödecke Research Prize in 1975, the Innovation Prize of Hessian Minister Economy in 1991, the W Warner Prize for Cancer Research in 1992, and the Colgate Research Prize in 2001. He holds membership of the European Society for Analytical Cellular Pathology and was their President from 1994 to 1997. He is a member of the Education Council of the International Academy of Pathology, German Division, since 1994, and a fellow of the International Academy of Cytology. 